\title{
High-peak-power femtosecond pulse generation using graphene as saturated absorber and dispersion compensator
}

CONFERENCE PAPER · JANUARY 2013

DOI: $10.1049 /$ cp.2013.1409

READS

9

8 AUTHORS, INCLUDING:

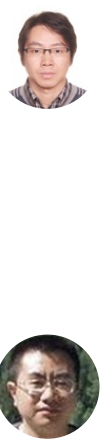

Lilin Yi

Shanghai Jiao Tong University 107 PUBLICATIONS 777 CITATIONS

SEE PROFILE

\section{Weisheng $\mathrm{Hu}$}

Shanghai Jiao Tong University 438 PUBLICATIONS 1,835 CITATIONS SEE PROFILE

\section{Zhengxuan Li}

Shanghai Jiao Tong University

24 PUBLICATIONS 97 CITATIONS

SEE PROFILE 


\title{
High-Peak-Power Femtosecond Pulse Generation using Graphene as Saturated Absorber and Dispersion Compensator
}

\author{
Lilin $\mathrm{Yi}^{1}{ }^{1}$, Zhengxuan $\mathrm{Li}^{1}$, Ran Zheng ${ }^{1}$, Zhenhua $\mathrm{Ni}^{2}$, Haiyan $\mathrm{Nan}^{2}$, Zheng Liang ${ }^{3}$, Rong Ding ${ }^{3}$, and \\ Weisheng $\mathrm{Hu}^{1}$ \\ ${ }^{1}$ The State Key Lab of Advanced Optical Communication Systems and Networks, Shanghai Jiao Tong \\ University, Shanghai 200240, China, lilinyi@sjtu.edu.cn \\ ${ }^{2}$ Department of Physics, Southeast University, Nanjing, China \\ ${ }^{3}$ Graphene research and characterize center, Taizhou Sunano New Energy Corporation, Taizhou, \\ China
}

\begin{abstract}
We demonstrate a passively mode-locked Erbium-doped fibre laser by using CVD fabricated graphene as both saturated absorber and dispersion compensator to achieve a 12-nJ energy pulse with 303-fs width, resulting in a 40-kW peak power, which is the highest value for graphene-based Erbium-doped passively mode-locked all-fibre laser.

\section{Introduction}

High-energy and high-peak-power ultrashort optical pulse has widespread applications in nonlinear optical processing, therefore has been addressed much attentions. Passive mode-lock shcheme in fibre laser is the main way to generate such a pulse. Graphene has been widely used in passively mode-locked fibre laser due to its well-known outstanding saturated absorption performance ${ }^{1}$. The graphene could be made by solution-based route, mechanically exfoliate or chemical vapor deposition (CVD) fabrication, which can be transferred to the fibre end, polished surface of D-shape fibre or the taped fibre waist to achieve light-graphene interaction. Both solution-based graphene transferred to D-shape fibre ${ }^{2}$ and CVD fabricated

mode-locked Erbium doped fibre laser using single-layer graphene as both saturated absorber and dispersion compensator. The graphene layer is CVD fabricated and is transferred onto the polished surface of a D-shape fibre. A dispersion-managed soliton with as high as 12-nJ energy, 40-kW peak power and 303-fs pulse width is achieved and no pulse breaking was observed. No other dispersion compensation devices are used except for the graphene, therefore reducing the complexity of the high-energy and high-peak-power fibre laser. Both the energy and peak power are the highest values for graphene-based Erbium-doped passively mode-locked all-fibre laser. This fibre laser is very promising in nonlinear optical processing field.
\end{abstract} graphene transferred to fibre end ${ }^{3}$ are used as saturated absorber to achieve high-energy and high-peak-power ultrashort pulse, where $7.25 \mathrm{~nJ}$, 4.87 kW pulse with 1.3-ps width and 7.3 nJ, 17.6 kW pulse with 415-fs width are achieved respectively. Generally speaking, graphene interacted with the evanescent filed is prefered due to its higher damage power compared to the perpendicular injection case. And to acquire a high energy pulse, long cavity and high pump power are required. Therefore graphene transfered onto D-shape fibre operating in a long-cavity fibre laser is a good choice for high-energy pulse generation. However, long cavity always results in a wide pulse, which is opposed to the requirement of a high peak power. To balance the contradiction and obtain a solition with both high energy and short width, dispersion compensation solutions such as Erbium-doped fibre (EDF) with negative disperison is employed in the long cavity to compensate the positive dispersion of single-mode fibre (SMF) ${ }^{4}$, which increases the complexity of the laser design.

In this paper, we report on the achievement of a high-energy and high-peak-power passively

\section{Experiments}

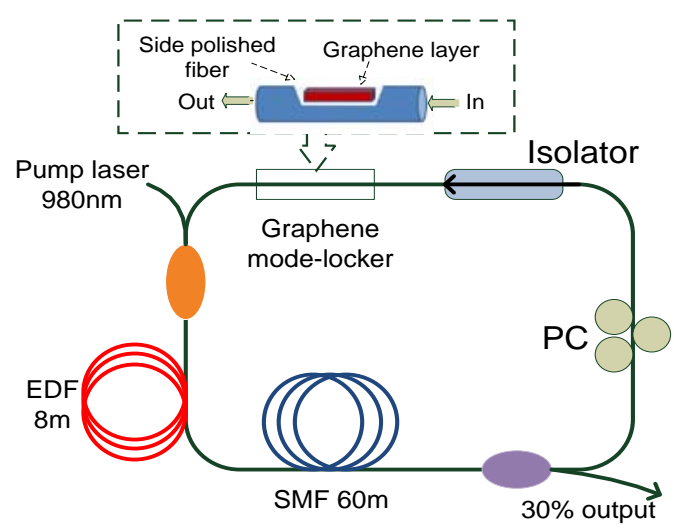

Fig. 1 The experiment setup

The graphene layer is made using CVD method, and then transferred onto the polished surface of a D-shape fibre. The length of the polished area is around $2 \mathrm{~cm}$. The inset of Fig. 1 shows the schematic of the graphene covered D-shape fibre. To increase the light interaction with the graphene, the D-shape fibre is deeply polished and the insertion loss without graphene is measured to be $7 \mathrm{~dB}$. In the previous work [2], the insertion loss of the D-shape fibre is only 1 
$\mathrm{dB}$, therefore the light-graphene interaction is very weak, which could be the reason why only picosecond pulse was achieved. After transferred the graphene to the D-shape fibre, the insertion loss is increased to $13 \mathrm{~dB}$. By connecting the graphene-covered D-shape fibre into an Erbium-doped fibre laser, passively mode-lock could be achieved. The configuration of the fibre laser is shown in Fig. 1, where the EDF length is $8 \mathrm{~m}$ with a group velocity dispersion (GVD) of $10 \mathrm{ps} / \mathrm{nm} / \mathrm{km}$, and the single mode fibre (SMF) length is $60 \mathrm{~m}$ with a GVD of $17 \mathrm{ps} / \mathrm{nm} / \mathrm{km}$. The total cavity length is $83 \mathrm{~m}$ including the fibre pigtail. There is no normal dispersion fibre employed in the cavity. A polarization-independent isolator is used to force the uni-directional transmission of the laser. A polarization controller is used to optimize the polarization state of the laser cavity and the 30\% port of a 30/70 coupler is used to export the high energy pulse.

\section{Results and Discussions}

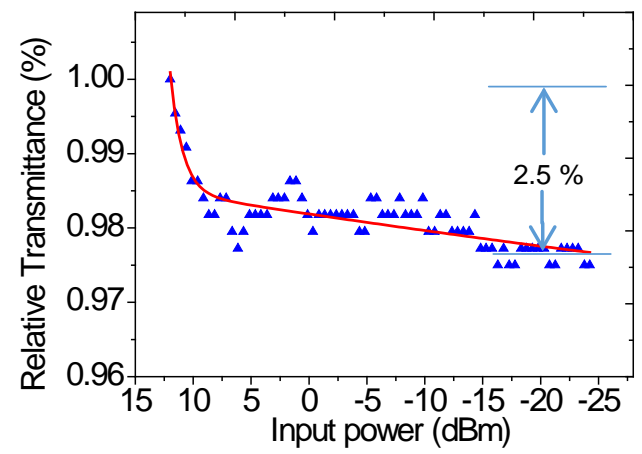

Fig. 2 Modulation depth measurement of the graphene covered on the D-shape fibre using our home-made passively mode-locked fibre laser as laser source.

We made two samples of graphene covered on D-shape fibre. We first used one sample as saturated absorber to make a passively mode-locked fibre laser, and used it as laser source to measure the saturated absorption property of the CVD fabricated graphene. The home-made fibre laser is with a repetition rate of $2.5 \mathrm{MHz}$ and pulse width of $320 \mathrm{fs}$. With the increase of the pulse power, absorbance of the graphene starts to saturate and the measured modulation depth is around $2.5 \%$. There was seldom report about the modulation depth measurement for the graphene interacted with the evanescent filed case and most of the measurements were done for the graphene transferred to the fibre end case.

Then we used the graphene with verified modulation depth as the saturation absorber to form a passively mode-locked fibre laser and investigated its performance. By optimizing the polarization state, the mode-locking operation can be achieved. The optical spectrum of the mode-locked fibre laser is shown in Fig. 3. The small peak around $1530 \mathrm{~nm}$ is attributed to the strong amplified spontaneous emission (ASE) power for short EDF length and the high cavity loss. The mode-locked pulse spectrum has a 3-dB width of $11.54 \mathrm{~nm}$, and is exactly fitted with the Gaussian profile, where a small shift between the two curves is due to the presence of 1530-nm peak on the mode-locked optical spectrum. The exact Gaussian fitting of the mode-locked spectrum proves the pulses are dispersion-managed solitons and the cavity dispersion is near to zero ${ }^{5}$. However, both SMF and EDF are anomalous dispersion fibre and there are no normal dispersion components in the cavity except for the graphene covered D-shape fibre, so we suppose the normal dispersion comes from the graphene covered $D$-shape fibre. The D-shape fibre itself is probably attributed to part of normal waveguide dispersion since the side polish changes the waveguide geometry, but its value is quite small and cannot compensate the accumulated dispersion of more than $80-\mathrm{m}$ anomalous fibres. Therefore the graphene should attributes to most of the normal material dispersion. Actually there has been report that CVD fabricated graphene exhibits strong normal dispersion but with no clear evidence and measurement results ${ }^{6}$. Here we prove its normal dispersion property and show its applications in dispersion management to achieve a dispersion-managed soliton, therefore no special EDF with normal dispersion is required as in [4] and [5].

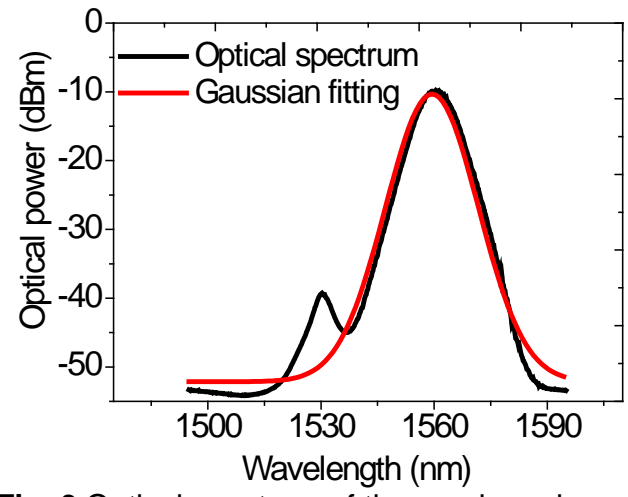

Fig. 3 Optical spectrum of the graphene based fibre laser

The measured output pulse train using low-speed oscilloscope and autocorrelation trace of the femtosecond pulse using FROG are shown in Fig. 4. The repetition rate of the fibre laser is around $2.5 \mathrm{MHz}$ and the measured pulse width is $303 \mathrm{fs}$. Although the fibre cavity is quite long, since the dispersion is well managed by graphene covered D-shape fibre, the ultra-short femtosecond pulse can still be achieved, which is different with the traditional high-energy fibre laser where long cavity results in strong chirp of 
the pulse therefore strict fibre length design is required outside the mode-locked fibre laser to de-chirp the pulse for ultra-short pulse and high peak power generation. The well dispersion management of the laser cavity also guarantees the single pulse generation and no pulse breaking was observed even at high pulse energy and peak power. The measured spatial chirp of the femtosecond pulse is as low as 0.0001 and the time bandwidth product is 0.415 , the phase variation of the pulse is also very minor, which verify the good quality of the femtosecond pulse.

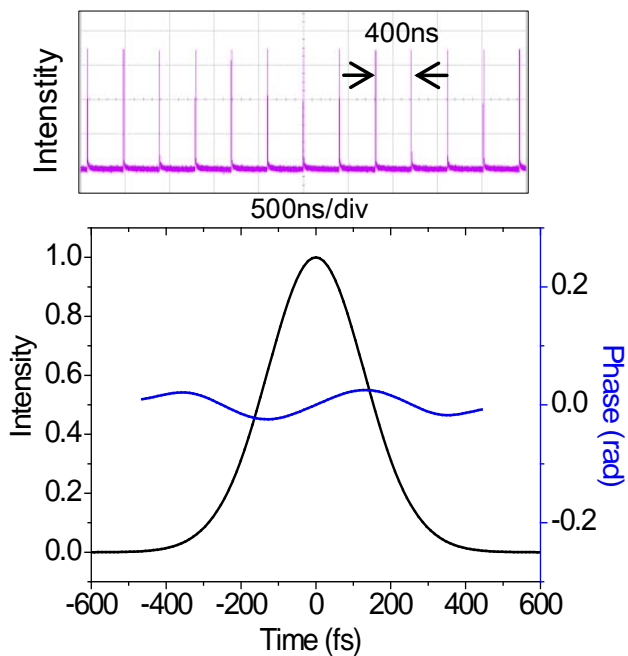

Fig. 4 Output pulse train and the autocorrelation trace of the femtosecond pulse

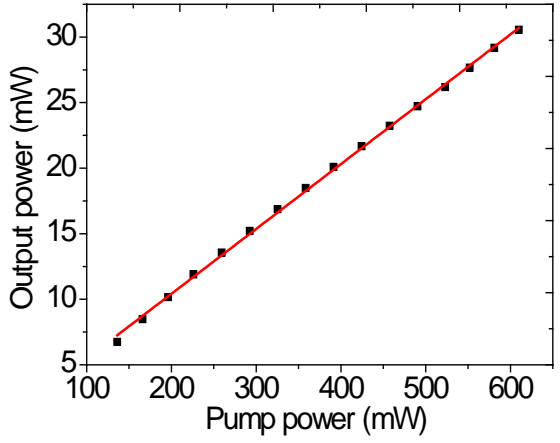

Fig. 5 The output power of the graphene based femtosecond fibre laser versus the pump power.

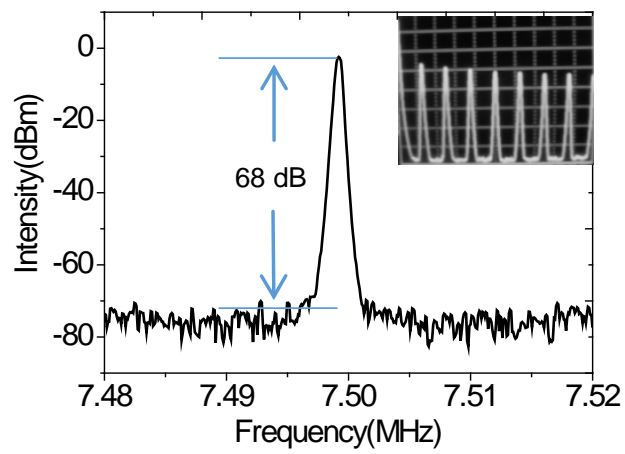

Fig. 6 The longitudinal-modes of the graphene based femtosecond fibre laser with $300-\mathrm{Hz}$ resolution.

We measured the averaged output power of the femtosecond pulse versus the pump power as shown in Fig. 5. The output power is almost linearly increased with the pump power. For the maximal available pump power of $610 \mathrm{~mW}$, the output power is around $30.4 \mathrm{~mW}$, corresponding to around $5 \%$ conversion efficiency. Note that the optical spectral shape and the pulse width keep unchanged with the increase of the pump power. We also measured radio-frequency (RF) spectrum of the mode-locked fibre laser as shown in Fig. 6 with a span of $40 \mathrm{KHz}$ and a resolution of $300 \mathrm{~Hz}$. The measured signal-to-noise (SNR) is as high as $68 \mathrm{~dB}$. The third peak rather than the fundamental peak is used to evaluate the SNR due to the high low-frequency noise of our used photodiode. The inset of Fig. 6 shows the RF spectrum up to 20 $\mathrm{MHz}$.

By considering the repetition rate of $2.5 \mathrm{MHz}$ and the pulse width of $303 \mathrm{fs}$, the calculated pulse energy and peak power are $12 \mathrm{~nJ}$ and 40 $\mathrm{kW}$ respectively, which are the highest values for graphene-based Erbium-doped passively mode-locked all-fibre laser. The conversion slope between the pulse energy, peak power and the pump power are $19.7 \mathrm{~nJ} / \mathrm{W}$ and 65.6 $\mathrm{kWN}$. By increasing the pump power, the pulse energy and peak power can be further improved.

\section{Conclusions}

By utilizing the saturated absorption and normal dispersion properties of graphene, a dispersion-managed soliton femtosecond fibre laser has been demonstrated. Attributed to the well dispersion management, ultra-short pulse generation in long cavity is achieved. The pulse energy and peak power are $12 \mathrm{~nJ}$ and $40 \mathrm{~kW}$ respectively, which are the highest values for all the graphene-based passively mode-locked Erbium-doped all-fibre lasers. The proposed fibre laser is supposed to have promising applications in nonlinear optical processing and other scientific research fields.

\section{Acknowledgements}

This work was partly supported by Nature Science Foundation China (61007041), Program of Shanghai Chen Guang Scholar (11CG11) and Program of Excellent PhD in China (201155).

\section{References}

[1] Z. Sun, D. Popa, T. Hasan et. al., Nano. Res. 3, 653, 2010.

[2] Y.W. Song, S.Y. Jang, W.S. Han, and M.K. Bae, Appl. Phys. Lett. 96, 05112, 2010.

[3] H. Zhang, D.Y. Tang, L.M. Zhao, Q.L. Bao, K.P. Loh, Opt. Exp. 17, 17630, 2009.

[4] D.popa, Z. Sun, F. Torrisi, T. Hasan, F. Wang, A.C. Ferrari, Appl. Phys. Lett. 97, 203106, 2010.

[5] H. Zhang, D.Y. Tang, L.M. Zhao et. al., Laser Phys. Lett. 7, 591, 2010.

[6] Q. Bao, H. Zhang, W. Wang, Z. Ni et. al., Adv. Funct. Mater. 19, 3077, 2009. 